Chapman University

Chapman University Digital Commons

Psychology Faculty Articles and Research

Psychology

2012

\title{
Self-Organizing Biopsychosocial Dynamics and the Patient-Healer Relationship
}

David Pincus

Chapman University, pincus@chapman.edu

Follow this and additional works at: http://digitalcommons.chapman.edu/psychology_articles

Part of the Alternative and Complementary Medicine Commons, Health and Medical Administration Commons, and the Medical Humanities Commons

\section{Recommended Citation}

Pincus, D. (2012). Self-organizing biopsychosocial dynamics and the patient-healer relationship.Research in Complementary Medicine 19(1), 22-29. doi: 10.1159/000335186

This Article is brought to you for free and open access by the Psychology at Chapman University Digital Commons. It has been accepted for inclusion in Psychology Faculty Articles and Research by an authorized administrator of Chapman University Digital Commons. For more information, please contactlaughtin@chapman.edu. 


\section{Self-Organizing Biopsychosocial Dynamics and the Patient-Healer Relationship}

\section{Comments}

This is a pre-copy-editing, author-produced PDF of an article accepted for publication in Forschende Komplementärmedizin/Research in Complementary Medicine, volume 19, supplement 1, in 2012 following peer review. The definitive publisher-authenticated version is available online at DOI:10.1159/000335186.

\section{Copyright}

Karger 
From: Pincus, D. (2012). Self-organizing biopsychosocial dynamics and the patient-healer relationship. Forschende Komplementarmedizin (Research in Complementary Medicine), 19 (supplement 1). DOI: 10.1159/000335186.

Title:

Self-organizing Biopsychosocial Dynamics and the Patient Healer Relationship

\section{Authors \& Affiliations:}

David Pincus

Crean School of Health and Life Sciences, Chapman University, Orange, CA, 92866, USA

Address correspondence to David Pincus, Psychology, Chapman University, One University Drive, Orange, CA 92866; pincus@chapman.edu

Key Words: Self-organization; Non-linear Dynamical Systems; Complementary and Alternative Medicine; Empathy; Telomeres; non-locality; quantum physics; retrocausality 


\begin{abstract}
The patient-healer relationship has an increasing area of interest for complementary and alternative medicine (CAM) researchers. This focus on the interpersonal context of treatment is not surprising, as dismantling studies, clinical trials and other linear research designs continually point toward the critical role of context and the broadband biopsychosocial nature of therapeutic responses to CAM. Unfortunately, the same traditional research models and methods that fail to find simple and specific treatment-outcome relations are similarly failing to find simple and specific mechanisms to explain how interpersonal processes influence patient outcomes. This paper presents an overview of some of the key models and methods from nonlinear dynamical systems that are better equipped for empirical testing of CAM outcomes on broadband biopsychosocial processes. Suggestions are made for CAM researchers to assist in modeling the interactions among key process-dynamics interacting across biopsychosocial scales: Empathy, intra-psychic conflict, physiological arousal, and leukocyte telomerase activity. Finally, some speculations are made regarding the possibility for deeper cross-scale information exchange involving quantum temporal non-locality.
\end{abstract}


Linear Research into Interpersonal Dynamics: Cutting wood with a hammer

Complementary and alternative medicine (CAM) research has increasingly turned to the practitioner-patient relationship as a significant contextual factor involved in the healing process. This attention appears warranted for a number of reasons. First, despite the demonstrated effectiveness of various CAM procedures for a range of biopsychosocial outcomes placebo treatments that approximate the relational context of active treatments can produce nearequivalent outcomes [1]. Second, dismantling studies have demonstrated significant step-wise improvements in outcomes of CAM interventions that may be empirically linked to variation in empathic attunement between healers and patients [1-4].

This inability to dismantle specific therapeutic effects from well-designed placebos along with the clear cut evidence linking empathic attunement to outcomes begs the comparison between CAM and with the broader literature from psychotherapy [1] and behavioral medicine [5]. If the empirical outcomes of random clinical trials (RCT's) and dismantling studies continue down the road of psychotherapy research, the end result will be an inability to determine differential efficacy across CAM treatments, an inability to clearly tie outcomes to the "active ingredients” of treatment, and moderate but consistent effects across treatments that are directly attributable to qualities of the healer-patient context [6].

When viewed through the lens of linear research, one may interpret such evidence as indicating that specific healing procedures in CAM are lacking in efficacy, or worse irrelevant. If placebo procedures are equivalent to active treatments, and relational factors such as empathic attunement are the predominant predictors of outcome across conditions in double-blind RCT's, then the evidence at first glance would suggest the abandonment of such specific procedures and a focus on enhancing the positive effects of the healing context.

An alternative explanation for such effects, however, lies simply in the possibility that these outcomes are the result of a modeling problem, rather than a problem of treatment or data namely the use of simple linear models to understand phenomena that are highly complex and 
non-linear. Using linear models to study interpersonal dynamics is like cutting wood with a hammer. It can be done, but only with great effort; and the wood will be ruined in the process. In the same way, models aimed at identifying simple, independent, and proportional causes in CAM treatments are likely to produce little more than a mess of useless variance.

Indeed, CAM treatments are not alone in running against tough competition in RCT's, particularly when the clinical condition is complex (e.g., caused by multiple biopsychosocial factors) and chronic. For example, a recent outcome trial for irritable bowel syndrome [7] demonstrated that placebo pills produced significant improvements even when patients were informed that the pills they were taking were inert and the pill bottles were labeled "Placebo." Such perplexing results are not so perplexing when one realizes that the actual healing processes are entirely left out of such RCT's. What happens at the social, individual, biological and chemical scales when an open label placebo is prescribed to an individual? How do change processes propagate across these scales? What does healing from IBS even mean in within the context of a highly interactive biopsychosocial system?

RCT's, dismantling studies, and traditional regression models each are predicated upon the assumptions of the general linear model. As such, they are well suited to phenomena that meet a set of linear and reductionist assumptions: (1) Cause effect relationships are proportional; (2) Causes are independent with additive effects; and (3) error variance is independently and identically distributed (IID). Nonlinear research models, by contrast, allow phenomena to have multiple and complex causes, with error components containing varying degrees of determinism. For example, such interconnected complex adaptive systems may display momentum effects and systemic memory over time, with outcome events organized as probability density functions that are inverse power-laws (IPL's; fractal Pareto distributions) rather than bell-shaped (normal Gaussian distributions). Models involving self-organization and emergence are key examples, where a sufficient number of systemic components with sufficient interconnections produce holistic order, capable of self-regulation over time. Methodologies from NDS allow for the 
measurement of global complexity in self-organizing relationship dynamics over time, as well as the ways that part-whole interactions across scales impact health [8].

For example, it has been demonstrated that the experimental induction of personality conflict within an individual leads to decreased complexity of fractal complexity in group dynamics, while conflict resolution leads to renewed flexibility [9]. Essentially, interpersonal systems shed flexibility in their fractal (i.e., across scales) branching patterns when a part of the system (i.e., a group member) becomes unhealthy [6]. Furthermore, empirical tests of fractal patterning in group behavioral dynamics lead to the emergence of relational qualities including closeness, control and conflict, and that these relational qualities simultaneously constrain subsequent behavioral dynamics unfolding over time - a cross-scale process of circular cause that is the hallmark of self-organizing systems [10]. Furthermore, nonlinear modeling of physiological arousal patterns over time have demonstrated that individuals engaged in simple conversations exchange entropy in these patterns above and beyond simple influence in ups and downs [11]. These, and other initial results, using NDS models to study physiology, personality and small group dynamics suggest that biopsychosocial systems are "sticky," meaning that linkage via complex, multi-scale constellations of information exchange is the norm $[6,8,12]$.

Indeed, a key factor in the typical 2 to 1 advantage in variance accounted for by nonlinear models over their linear alternatives in empirical studies [13] lies in their ability to account for deterministic varieties of noise (see Bell et al., this issue for more on this topic). Indeed, many health and interpersonal situations studied using NDS models explicitly focus upon changes in the levels of noise produced by a system, rather than treating noise as a statistical nuisance to be minimized to allow for a better canvas upon which to view simple differences in means. For example, research using orbital decomposition (OD) $[14,15]$ has tested the fit of fractal, IPL, distributions on the interpersonal exchange dynamics over time in more than 30 groups thus far, including family interactions [15, 16], group therapy [10] and experimentally created groups [9]. In each case the dynamics conformed to the IPL, most often with $\mathrm{R}^{2}$ values greater than .90 . The normal curve has nothing to do with interpersonal dynamics. Relationships are emergent 
phenomena, not normal, and trying to focus on means and reduce "error" is literally like trying to understand a tree by cutting off its branches.

When one dispenses of the errant assumptions of linear models, the role of the patienthealer relationship may be viewed not as a competing explanation for the cause of healing in CAM, but rather as an entrance point for the introduction of some general non-linear models and empirical methods that may be more far more appropriate than linear approaches for determining the underlying causal processes involved in health and healing in general, and particularly for CAM interventions due to their grounding in holism. It is important to take a balanced view, however. Linear research in general and RCT's in particular are without utility in CAM research. This sort of either-or reasoning mistakenly oversimplifies the situation, and erroneously elevates ones models to a status above the phenomenon of interest. Models are simply tools, neither good nor bad, but rather more or less useful given the specific research question one is asking and the nature of the phenomenon in question. Rather, nonlinear dynamics provides some additional theoretical lenses that one may use to conceptualize the role of interpersonal dynamics within a holistic model of CAM intervention toward health and healing. Most importantly, nonlinear dynamics provides some broader empirical methods above and beyond the subset of linear models, which may be used to test specific hypotheses.

\section{Biopsychosocial dynamics}

Pincus \& Metten [10] have proposed a theory of biopsychosocial processes grounded in self-organization theory, a potentially useful framework for NDS studies in CAM involving interpersonal processes. Essentially, their theory suggests that physiological components (e.g., respiration, movement, cardiovascular, and nervous system) are linked within a self-organizing network resulting in emergent nodes at the higher level psychological scale (e.g., habits, traits, self-aspects) that are likewise linked, self-organizing and emergent into the social scale (e.g., interpersonal closeness, control and conflict). Grounded within this notion of biopsychosocial dynamics within a hierarchical, self-organizing, complex adaptive system (CAS) they define resilience as: "the meta-flexibility of the system: the ability to respond to a perturbation by 
either becoming rigid and robust, or flexible and fluid without becoming stuck or falling apart respectively.” Generally speaking, short-term increases in coupling-strength or coordination among nodes within the biopsychosocial networks hypothetically acts as an adaptation toward maintaining systemic integrity in the face of turbulent flows of information from within or without the system. By contrast, prolonged activation of this robustness reaction is thought to lead to a loss of resilience through systemic breakdowns, tears in the fabric of the network structure - such as decoupling of cardiovascular dynamics from respiration at the level of physiology, or social isolation at the interpersonal scale.

The hypothalamic-pituitary-adrenal (HPA) axis is considered to be a central hub within this system, with a functional role of quick and efficient information transfer among the three scales: physiological, psychological, and social. Indeed, viewing the adaptive function of HPA axis as primarily structural provides a parsimonious account of otherwise disparate seeming processes such as Cortisol action on respiration, blood pressure and musculoscellatal dynamics, attention focus and cognitive biases, and stereotypic survival-based reflexes involving fight-orflight or tend-and-befriend [17 - Taylor*]. The various biopsychosocial responses to HPA activation involve a loss of free space of motion, and increase in coordination, and a loss of intentionality in preparation for more automatic and survival-based behaviors.

Holistic healing of the nature typically described in CAM would theoretically be thought to enable re-connection and repair among ruptures in the biopsychosocial networks that have been overwhelmed by prolonged HPA activation and other paradoxical effects of faulty attempts at protection and healing. This self-organizing biopsychosocial network theoretical framework also provides a good fit with CAM diagnostic systems that view symptoms not as phenomena to be suppressed through exogenous agents (i.e., allopathic medications), but rather as a unique set of functional response that can used to understand the underlying loss of resilience that may be targeted for healing via reconnection. Similarly, the typical progression backward temporal progression through the patient's series of symptoms along with 
accompanying HPA activation appears consistent with a model of biopsychosocial reconnection as the undergirding of renewed health and resilience [18].

Of most importance for the advancement of CAM research, this theory is empirically testable through a wide range of quantitative NDS methodologies. A full description of these methods is available elsewhere [10]. Yet conceptually the techniques are research strategies are rather simple, organized around four different levels of modeling: (1) direct modeling of the networks themselves; (2) time series techniques to capture aspects of complexity in particular system output(s); (3) state-space grids or orbital decomposition to assess interactions among several system components over time; or (4) a cusp catastrophe model (see figure 1).

\section{Insert Figure 1 about Here}

The cusp catastrophe is the most frequently studied response surface in the behavioral and social sciences [19]. Part of the cusp response surface is has a smooth path between extreme values on the order parameter (i.e., behavioral output of the system). This portion of the response surface is characterized by a linear relationship between one of the control parameters, the asymmetry parameter, and the order parameter. This linear relationship in health may be thought of as representing the traditional biomedical disease model, where exposure to some pathogen may be thought to produce a linear outcome on severity of illness. Or one may conceptualize this part of the response surface as a linear dose response relationship between some medication and a decrease in symptoms.

The nonlinear effect of the cusp catastrophe is moderated by interaction of the asymmetry parameter with a second control parameter known as the bifurcation parameter. As the influence of the bifurcation parameter is increased, the boundary between health and illness becomes increasingly discontinuous (see Figure 1). Pincus and Metten [10] suggest that increasing bifurcation as such may be used as an index of disintegration among the underlying biopsychosocial system, a loss of systemic resilience. Prolonged HPA activation, allopathic 
stress [20] would be a good candidate for modeling this bifurcation in health research. When the bifurcation parameter is high, the system tends to get stuck in one of two regions, one described as pseudo-health - where there is a lack of symptoms masking poor underlying health and resilience, and another region involving stuckness in a chronic and multifaceted condition of illness (i.e., complex illness) - the type of health status that is non-responsive to allopathic interventions and in which CAM practitioners tend to do their work (see Table 1).

\section{Insert Table 1 about Here}

Finally, when the system is near the cusp point, the system will display a toggling backand-forth between the two discrete states of health (i.e., pseudo-wellness and complex illness, see Figure 1). This dynamic is well known dynamics is referred to as hysteresis, and may be another indicator of characteristic of the process of symptom re-experiencing, worsening, and reversal that is often observed in positive responses to CAM interventions [18; see also Koithan et al this issue].

It is important to note that each of these modeling strategies and methodologies are simply tools to allow one to test various hypotheses arising from the notion that biopsychosocial systems are hierarchically emergent and self-organizing. They are not mutually exclusive or theoretically contradicting, and indeed may be overlaid upon one another rather seamlessly within the context of a single theory of biopsychosocial network dynamics (i.e., Figure 1).

\section{Arousal, Telomeres, and Quantum coherence: A possible future for integrated health science}

The theory of biopsychosocial self-organization outlined above suffers from being nonspecific. What are the most important network nodes at each scale? How exactly does information flow across scales signaling shifts toward and away from flexibility? How exactly do these nodes influence repair versus disintegration over time? Which levels are most important? And how far down the ladder of scale does the process go? This final section will 
(a) suggest some networked nodes for empirical focus at the social, psychological, and physiological scales; (b) describe the mechanisms of information transfer downward and upward through these nodes pertaining to structural coherence or de-coherence over time; and (c) provide a highly speculative yet empirically testable possibility that information from the quantum scale may play some role in recovery from complex illness.

At the social scale, researchers should continue to focus on empathy - following from roughly sixty years of investigation consistently linking empathic attunement to positive outcomes in psychotherapy, and some initial comparable results in CAM [4]. Researchers should be mindful to define empathy with precision, sticking as close to Roger’s [21] original definition: “To sense the client's private world as if it were your own, but without ever losing the 'as if' quality” (p. 829). The experience of being understood is central to the integrating function of empathy, whereby an individual settles into one's experience with acceptance, allowing for more flexible, self-consistent, and adaptive responses to whatever negative information is contained within that experience. As researchers focus on the healing dynamical effects of empathy on flows of interpersonal and self-experience, they may find it efficient to focus on contempt as the opposite process, whereby, whereby a valued other dismisses ones experience as invalid, with broad and complete judgment, and with a love withdrawal that embodies an acute sense of social isolation. Contempt tears away at the integrated aspects of one's social and selfexperience and activates the HPA-axis, increasing the likelihood of key biopsychosocial disintegrations and pockets of sustained rigidity and isolation. The experience of contempt by ones partner in marriage, for example, has been found to be linked to heart disease at levels on a par with smoking and poor nutrition [22].

Greenberg has extended the discussion of how empathy operates, suggesting that the imagination is central [23-24]. It is clear too that the imagination is the conduit for the spread of acceptance, non-judgment, and understanding by another to the same relational qualities with one's self, as well as a teleological orientation toward a future self as healed. When viewed in structural terms, biopsychosocial systems would be expected to become more flexible when 
judgment and struggle shift in the direction of: acceptance, non-judgment, and decreased automaticity in attempts at misguided and ineffective direct control strategies. When viewed in terms of the specific transfer of novel information to allow for such flexibility across psychosocial scales of a self-organizing network, the imagination should be the node at the psychological scale upon which to focus in empirical studies. For example, one could measure the dynamical or structural flexibility of key health, developmental, or self-concept related imagery over treatment and in response to either empathy or contempt [5]

Moving downward, past the global impacts of the HPA response on biological coherence, CAM researchers should focus their attention on the role of telomeres and its accompanying chemical messenger telomerase as a key process regulating the ongoing structural integrity of cell replication over time. Telomeres function as caps at the end of genes, allowing a stop-gap for messenger RNA during genetic transcription. When telomeres get shorter, errors in transcription are more likely at the ends of strands of DNA. When telomeres get longer, transcription is more exact, increasing dynamical genetic reproductive integrity. Telomere length at leukocytes has been found to be related to broadband physiological breakdowns (e.g., heart disease, cancer, and aging processes) due to stress from a variety of biopsychosocial sources [25]

Does biopsychosocial resilience involve information flows emerging from scales smaller than genes? Some recent, well-controlled experimental studies published recently in the prestigious Journal of Personality and Social Psychology by Bem suggest that shifts to quantum scale information flows could be linked to the HPA response [26]. These experiments provide strong evidence for retrocausal effects in cognition that are likely mediated by physiological arousal. Bem used well-known perceptual bias and memory paradigms, but with learning trials conducted after the test trials - reversing the order that any logical person would deem necessary for learning to take place. For example, participants were able to consistently beat chance levels in guessing where (right or left) an emotionally arousing image would appear on a computer screen when the images were presented at a later point in time. Moreover, this apparent 
retrocausal effect was entirely mediated by the personality trait of sensation-seeking (i.e., within the broader trait of extroversion); sensation seekers were around 57\% accurate in guessing these randomly generated positions. Bem and others [26-28] have suggested that some connection to non-local physics may be involved in explaining this effect, as retrocausal effects have been well-demonstrated at the quantum level and because conscious processes within observers are entangled with stochastic quantum effects [26-29]. Stapp has suggested that Bem's results might provide the beginning of a revision to the classical view of quantum mechanics whereby observer-universe entanglement occurs only in one direction - whereby the observer asks a question and the universe selects the response in a stochastic manner. Bem's results may suggest a two-way observer-universe interaction involving a shift from stochasticity on the part of the universe. Yet precisely how or why these deep cross-scale connections may occur, and how physiological arousal may be involved has remained theoretically illusive.

Given that sensation-seekers are known to be lower in baseline HPA axis arousal [30], and that these retrocausal effects occur only when using emotionally arousing stimuli, one might use a direct extension of Pincus \& Metten's biopsychosocial resilience model to suggest that the coherence inducing effects of physiological arousal could extend downward to the quantum scale. Specifically, arousal induced quantum entanglement effects may serve to shift the stochastic dynamics at the quantum scale from their usual background Gaussian noise to fattertailed probability distributions (e.g., Pareto distribution or IPL). Such a shift is consistent with the shifts that have been observed experimentally in distressed psychosocial systems [6, 9-10], and are a hallmark on coherence making dynamics via self-organization.

Below the level of telomeres and genes, it is possible that shifts in physiological arousal may also facilitate the transfer of useful information across time, allowing for some degree of teleological cause to come to bear in healing - for example imagery pertaining to future health may impact current healing. As far-fetched and ontologically difficult as this possibility may sound, it would be entirely consistent with the recent experimental effects obtained by Bem and by the more longstanding non-local quantum physics that have been used to interpret Bem's 
results. Furthermore, this suggestion that the imagination may impact health via arousal induced quantum to social coherence mechanisms is consistent with the positive role that physiological arousal plays in psychotherapy outcomes [31] , the connection between immersion and therapeutic effects in guided imagery for pain [5], and empathy moderated exchanges of physiological entropy observed during social interaction [11].

More important than plausibility, such arousal moderated retro-causal effects are empirically testable. For example, researchers in CAM could begin to look for healing responses to interventions that occur prior to the intervention. One good place to look for such evidence of these healing responses would be in telomerase activity on Leukocytes. Moderating variables to be tested should focus upon changes to physiological arousal in the patient, empathic attunement on the part of the therapist, and patterns of physiological entropy exchange between patient and therapist during the intervention. For example, an interested researcher could measure specific symptoms, empathic attunement, and telomerase levels prior to each treatment, just after each treatment, and at follow-up. During each treatment, physiological arousal of the patient and healer could be monitored over time - providing a measure for change in arousal by the patient during the procedure as well as entropy exchange between patient and healer. If interested, it might also be interesting to collect qualitative data pertaining to specific healing related images that occur spontaneously during treatment sessions within or between the patient and healer. Evidence for quantum-interpersonal retro-cause in healing would be provided by healing responses that occur prior to sessions involving sufficient patient arousal, high levels of entropy exchange in the healer-patient relationship, high levels of reported empathic attunement and sufficiently robust spontaneous images of future health emerging during sessions. Alternatively, arousal levels of the patient could be manipulated experimentally and examined for effects on telomerase increases, or telomere elongation occurring prior to treatment. 


\section{References}

1. Hyland, M.E.: A tale of two therapies: psychotherapy and complementary and alternative medicine (CAM) and the human effect. Clin Med 2005: 5; 361-367.

2. Kaptchuk TJ, Kelley JM, Conboy LA et al.: Components of placebo effect: randomized controlled trial in patients with irritable bowel syndrome. BMJ 2008: 336; 999-1003.

3. Gould A, MacPherson H: Patient perspectives on outcomes after treatment with acupuncture. J of Alt and Comp Med 2001: 3; 261-268.

4. Bikker AP, Mercer SW, Reilly D: A pilot prospective study on the consultation and relational empathy, patient enablement, and health changes over 12 months in patients going to the Glasgow homeopathic hospital. The J of Alt and Comp Med 2005: 11; 591-600.

5. Pincus D, Sheikh AA. Imagery for pain relief: a scientifically grounded guidebook for clinicians. New York: Routledge; 2009.

6. Pincus D. Coherence, complexity, and information flow: self-organizing processes in psychotherapy. In: Guastello SJ, Koopmans M, Pincus D, editors. Chaos and complexity in psychology: theory of nonlinear dynamical systems. Cambridge MA: Cambridge University Press; 2009.

7. Kaptchuk TJ, Friedlander E, Kelley JM, Sanchez MN, Kokkotou E, Singer JP, Kowalczykowski M, Miller FG, Kirsch I, Lembo AJ. Placebos without deception: A randomized controlled trial in irritable bowel syndrome. PLoS One. 2010; 5(12):1-7.

8. Pincus D, Metten, AM. Nonlinear dynamics in biopsychosocial resilience. Nonlinear Dynamics, Psychol Life Sci. 2010; 14:353-380.

9. Pincus D, Fox KM, Perez KA, Turner JS, McGee AR. Nonlinear dynamics of individual and interpersonal conflict in an experimental group. Small Gr Res. 2008; 39:150-178.

10. Pincus D, Guastello SJ. Nonlinear dynamics and interpersonal correlates of verbal turntaking patterns in group therapy. Small Gr Res. 2005; 36:635-677.

11. Guastello SJ, Pincus D, Gunderson P. Electro-dermal arousal between participants in a conversation: Nonlinear dynamics for linkage effects. Nonlinear Dynamics, Psychol Life Sci. 2006; 10:341-375.

12. Koithan M, Verhoef M, Bell IR, Ritenbaugh C, White M, Mulkins A. The process of whole person healing: "unstuckness" and beyond. J Altern Complement Med. 2007;13(6):65968.

13. Guastello SJ. Chaos, catastrophe, and human affairs: applications of nonlinear dynamics to work, organizations, and social evolution. Mahwah, NJ: Lawrence Erlbaum; 1995.

14. Guastello SJ. Orbital decomposition: Identification of dynamical patterns in categorical data. In Guastello SJ, Gregson RAM, editors. Nonlinear Dynamical Systems Analysis for the Behavioral Sciences: Real Data. Boca Raton, FL: CRC Press/Taylor \& Francis, 2010.

15. Pincus D, Ortega DL, Metten AM. Orbital decomposition for multiple time series comparisons. In Guastello SJ, Gregson RAM, editors. Nonlinear dynamical systems analysis for the behavioral sciences using real data. Boca Raton, FL: CRC Press/Taylor \& Francis; 2010. 16. Pincus D. A framework and methodology for the study of non-linear, self-organizing family dynamics. Nonlinear Dynamics, Psychol Life Sci. 2001; 5:139-174.

17. Taylor SE. Tend and befriend: Biobehavioral bases of affiliation under stress. Current Directions in Psychological Sci. 2006; 15(6):273-277. 
18. Zeff J, Snider P, Myers S. The hierarchy of healing: The therapeutic order. In The Textbook of Natural Medicine (3rd edition). Elsevier Publishing; 2006.

19. Guastello SJ. Managing emergent phenomena: Nonlinear dynamics in work organizations. Erlbaum; 2002.

20. McEwen BS, \& Wingfield, JC. The concept of allostasis in biology and biomedicine. Hormones and Behavior. 2003; 43:2-15.

21. Rogers $C$. The necessary and sufficient conditions of therapeutic personality change. J of Consulting and Clinical Psych. 1957; 60:827-832.

22. De Vogli R, Chandola, T, \& Marmot, MG. Negative aspects of close relationships and heart disease. Arch Intern Med. 2007; 1679180:1951-1957.

23. Greenberg LS. My change process: From certainty through chaos to complexity. In Goldfried M, editor. How Therapists Change: Personal and Professional Reflections (pp. 247270). Washington, DC: APA. 2000

24. Greenberg LS. Emotion-focused therapy: Coaching clients to work through their feelings. Washington, DC: APA. 2002.

25. Epel, ES. Telomeres in a life-span perspective: A new "psychobiomarker"? Current Directions in Psychological Sci. 2009; 18(1):6-10.

26. Bem DJ. Feeling the Future: Experimental Evidence for Anomalous Retroactive Influences on Cognition and Affect. J of Personality and Social Psych. 2011. doi: 10.1037/a0021524

27. Stapp, HP. Reason and Retrocausation. Paper presented at the AAAS conference, San Diego, CA, USA, June 13-16, 2011.

28. Kafatos, M., Nassikas, A.A. (2011) "Retro-causation, Minimum Contradictions and nonLocality”, Proceedings AAAS, San Diego.

29. Kafatos, M., Tanzi, R.E., Chopra, D (2011) "How Consciousness Becomes the Physical Universe”, Journal of Cosmology, 2011, Vol. 14. 1318-1328

30. Zuckerman M, \& Kuhlman DM. Personality and risk-taking: Common biosocial factors. J of Personality. 2000; 68(6):999-1029.

31. Carryer JR, \& Greenberg LS. Optimal levels of emotional arousal in experiential therapy of depression. J of Consult and Clin Psyc. 2010; 78(2):190-199. 


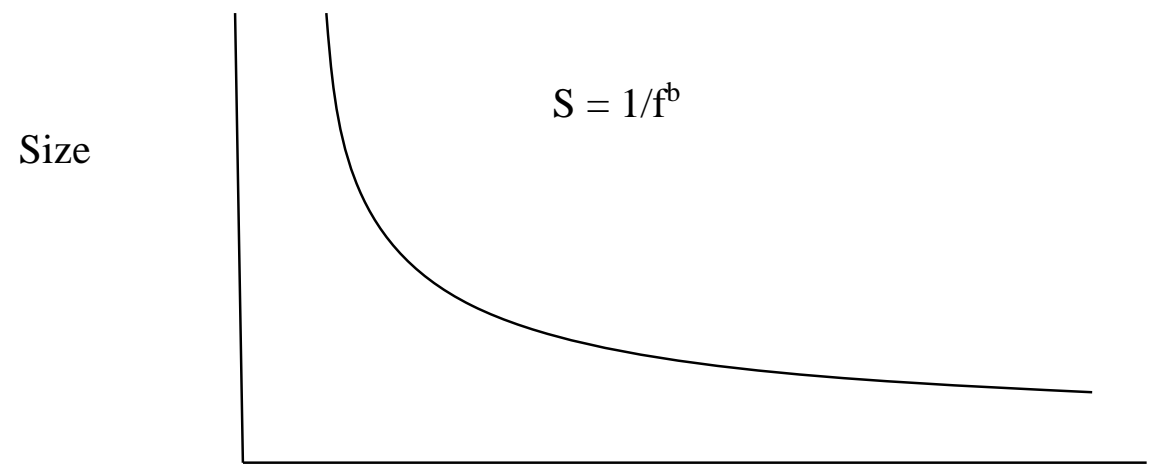

Frequency

Figure 1a. An Inverse Power Law (IPL), or Pareto distribution. Note the relationship between size (S) and frequency (f), which varies exponentially depending upon the shape of the curve (b), which may be used as an estimate of complexity (i.e., fractal dimension).

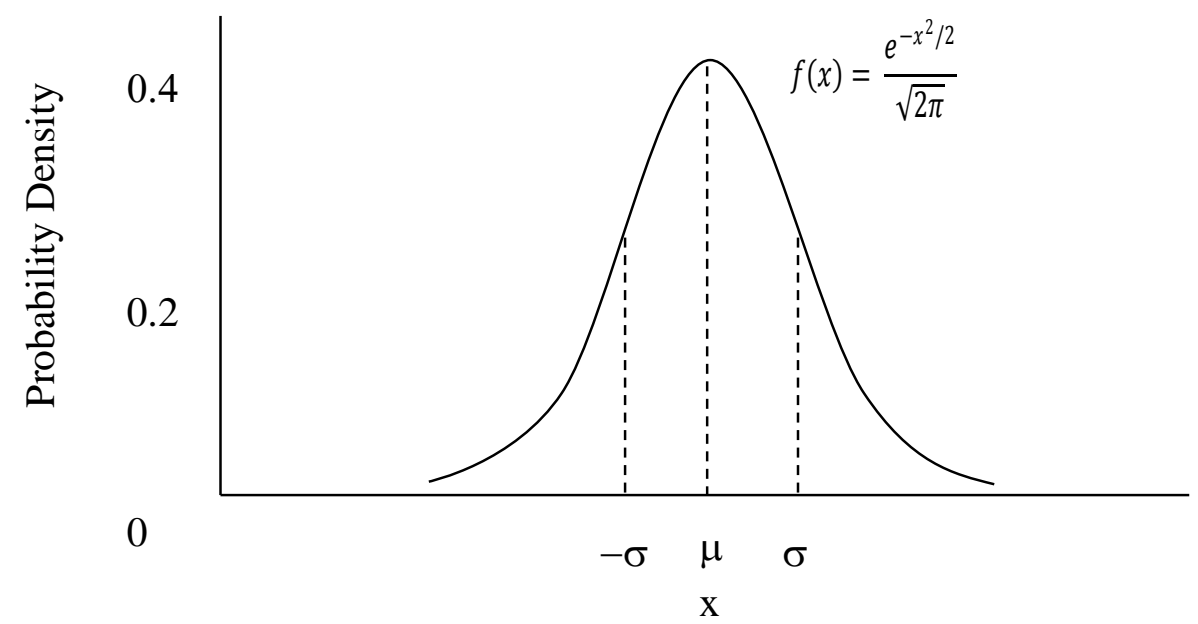

Figure 1b. A normal or Gaussian Distribution, where the mean $(\mu)=0$ and standard deviation $(\sigma)=1$. 


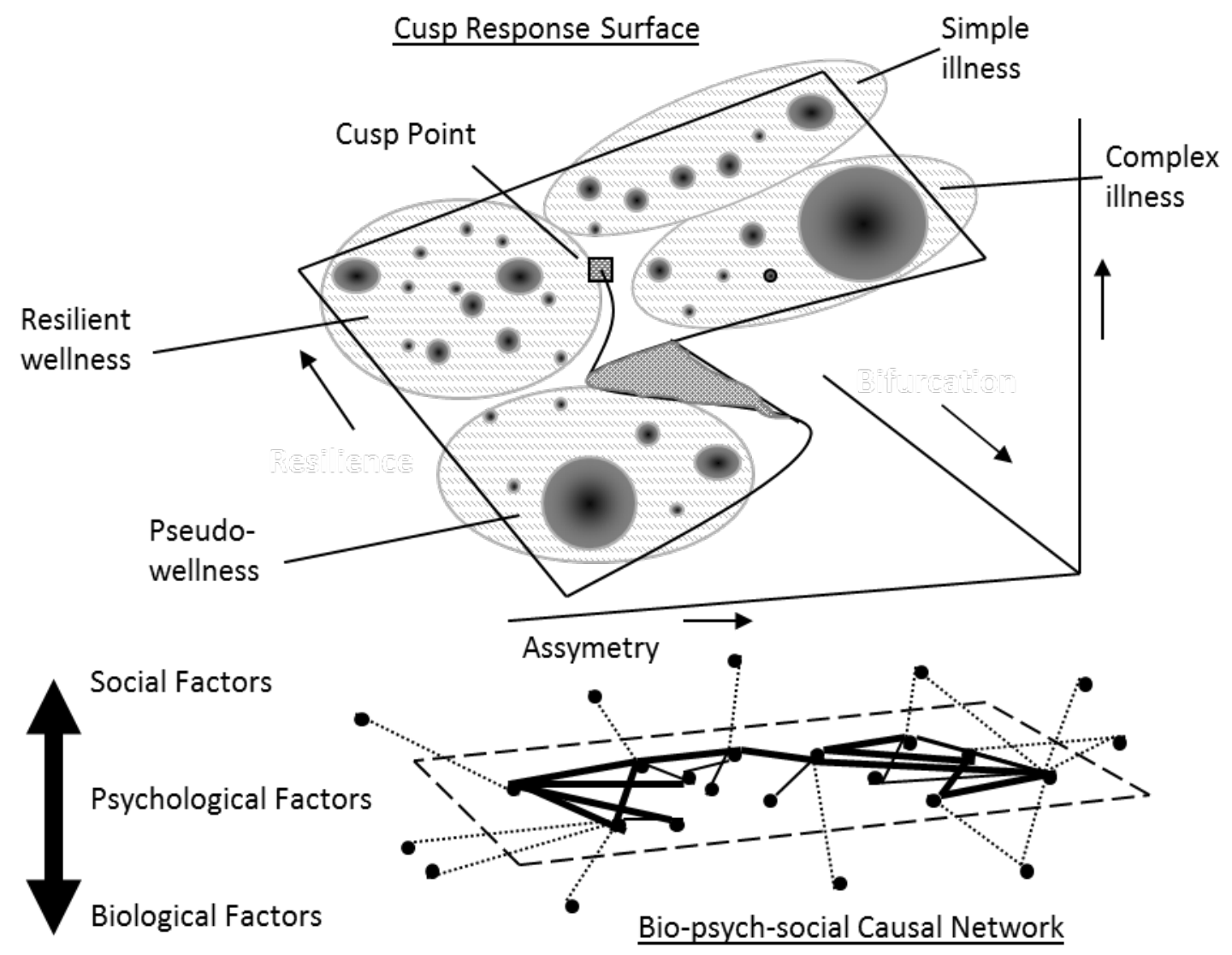

Figure 2. A superposition of different models of self-organization that may be used to describe and measure biopsychosocial resilience as well as four different states of health: Resilient wellness, Pseudowellness, Simple illness and Complex illness. Reprinted with permission from Pincus \& Metten (2010). 

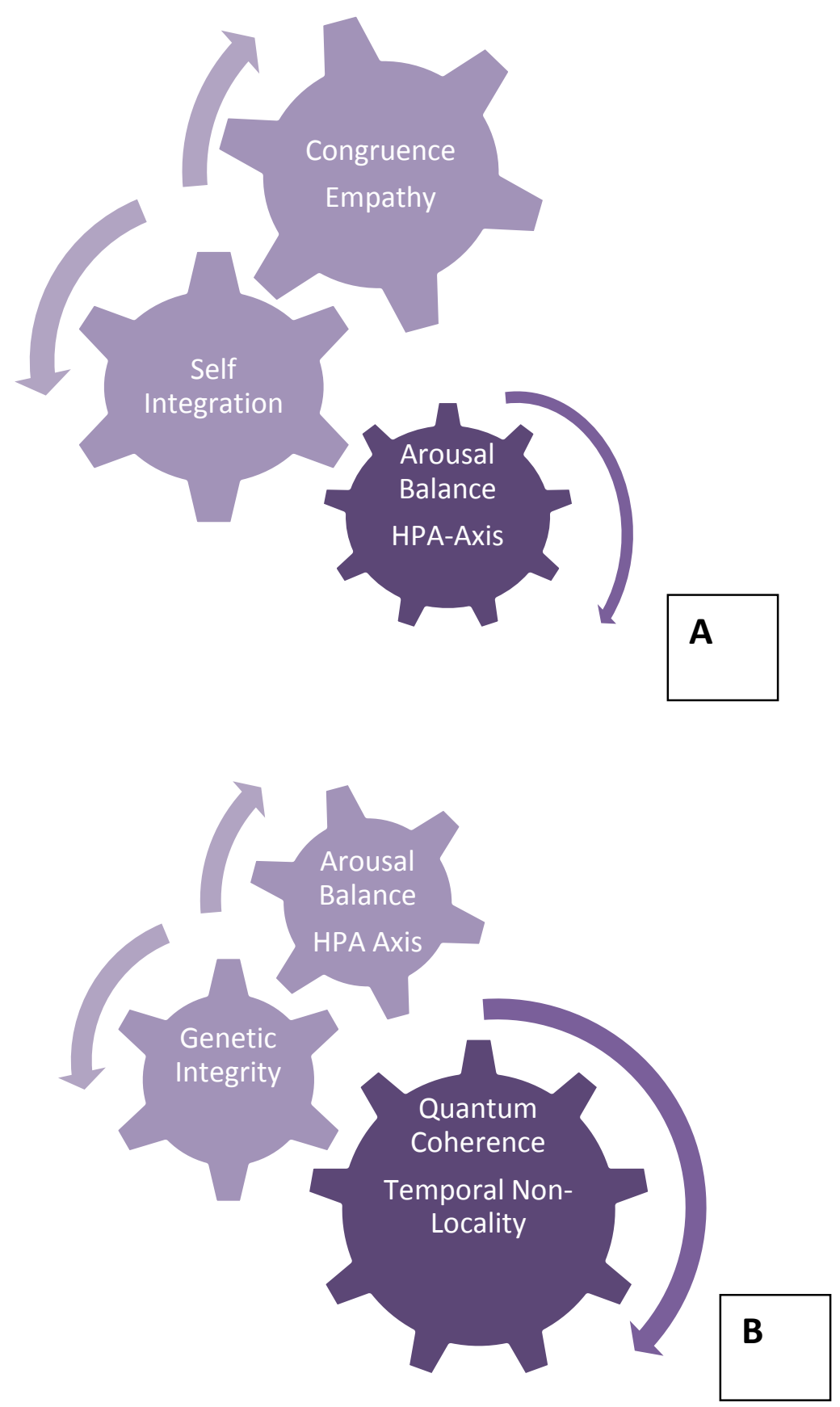

Figure 3a-b. a) Illustration of top-down information flow influencing spread of self-organized coherence, from empathy at interpersonal scale to flexible arousal regulation via HPA-axis. b) Hypothesized continuation of spread of coherence-creating influence of self-organization downward from HPA-axis through telomeres to influence genetic integrity in leukocytes, and further downward to potentially induce quantum coherence - shifts toward temporal non-locality allowing for healing information to travel backward in time. 